\title{
Euclidean Norm in Composite Severity Score to Evaluate an Allergic Reaction from Conjunctival Provocations
}

\author{
Anatoli Astvatsatourov ${ }^{1,2 *}$ and Ralph Mosges ${ }^{1}$ \\ ${ }^{1}$ Institute of Medical Statistics, Informatics and Epidemiology, University Hospital of Cologne, Lindenburger Allee 42,50931 Cologne, Germany \\ ${ }^{2}$ Cologne Ophthalmological Reading and Image Analysis Center, Experimental Ophthalmology, University Hospital of Cologne, Kerpener Str. 62 , 50937 Cologne, Germany
}

\begin{abstract}
A simple and robust estimation method is suggested to outline the severity of an allergic reaction from several conjunctival challenges combined in one diagnostic procedure - conjunctival provocation test (CPT). The CPT is performed by a stepwise application of an allergen solutions of increasing concentrations onto the patient's conjunctiva. The allergic reaction is estimated after each application by a numerical severity score. The severity scores are combined into one composite value, using their Euclidean norm divided by the square number of applications. The difference of the composite scores before and after the therapy is proposed as an appropriate outcome parameter in drug registration trials to represent the therapeutic effect. Applicability of the suggested method is shown in a randomized controlled double-blind dose-finding study of 150 patients with birch pollen allergy. The superior effect of the highest doses over the lowest dose for this study was not found to be statistically significant ( $p<0.1$ in U-test).
\end{abstract}

Keywords: Allergy diagnosis; Conjunctival provocation; Severity score; Composite score; Allergic rhinoconjunctivitis

\section{Introduction}

The conjunctival provocation test (CPT) is a diagnostic procedure for testing airborne allergens causing allergic rhinoconjunctivitis $[1,2]$. It has been thoroughly evaluated with regard to its precision [3] and compared with nasal provocation tests [4]. Nowadays, the CPT is used for evaluation of allergic rhinoconjunctivitis $[5,6]$ and is accepted by regulatory authorities as an outcome parameter in dose-finding trials.

The CPT consists of several conjunctival challenges with increasing allergen concentrations. Each challenge is an instillation of the ophthalmic solution containing an allergen. The conjunctiva allergic reaction is assessed after each challenge by the numerical score. If the score is equal or higher than the threshold value, the CPT stops. In the considered clinical study the allergic reaction to each challenge was assessed in terms of severity scores $0 \leq \mathrm{s}_{i} \leq 4$, by use of the Gronemeyer's grading scale $[4,7]$, presented in Table 1 . The CPT was performed with a maximum of three allergen challenges $(1 \leq i \leq 3)$. The used diluted allergen solutions are: 100, 1000 and 10000 SQ-E/ml ('ALK-lyophilized SQ', ALK Abell'o A/S, Hørsholm, Denmark) for first, possible second and third challenges, respectively.

\section{The CPT steps are:}

1 Before challenge, the right (control) eye is treated with a diluent without an allergen to reveal possible non-allergic reaction. If control reaction is positive, the patient is excluded from the study.

2 If control reaction is negative, a single drop of allergen solution with the lowest titration, $100 \mathrm{SQ}-\mathrm{E} / \mathrm{ml}$, is put into the left (provocation) eye. If the assessed severity score is $s_{1} \geq 2$, the allergic reaction is positive and the CPT is complete. Here, the number of challenges to reach a positive reaction is $n=1$.

3 If the allergic reaction to $100 \mathrm{SQ}-\mathrm{E} / \mathrm{ml}$ is negative (i.e. $\mathrm{s}_{1}<2$ ), the solution with the next higher concentration, $1000 \mathrm{SQ}-\mathrm{E} / \mathrm{ml}$, is applied. If $s_{2} \geq 2$, the positive CPT result is reached at $n=2$.

4 If the reaction to $1000 \mathrm{SQ}-\mathrm{E} / \mathrm{ml}$ results in $\mathrm{s}_{2}<2$, a drop with the highest concentration $10000 \mathrm{SQ}-\mathrm{E} / \mathrm{ml}$ is applied. If the CPT is negative to application of $10000 \mathrm{SQ}-\mathrm{E} / \mathrm{ml}$, the patient is excluded from the study. Otherwise the positive CPT result is reached at $n=3$.

When accessing the reactive threshold only, non-zero information derived from challenges at lower concentrations is lost. For an accurate comparison of the allergic severity before and after the treatment, one should take into account non-zero severity scores of lower challenges, where the threshold value is still not reached and some allergic symptoms are already obviously observed. Therefore, it is suggested to estimate the severity with the composite score.

\section{Method}

The CPT is considered to be positive and complete, if the severity score $s_{i}$ is equal or greater than the threshold value $s_{t h}=2$. Here, $i$ is the challenge number, $1 \leq i \leq 3$. The composite score $C_{j}$ is calculated for the $j$-th patient $(1 \leq j \leq \mathrm{N})$ as an Euclidean norm of severity scores $s_{i}$,

\begin{tabular}{|c|c|}
\hline $\begin{array}{l}\text { Severity } \\
\text { score }\left(s_{i}\right)\end{array}$ & Description of the severity \\
\hline 0 & Stage 0: no subjective or visible reaction \\
\hline 1 & Stage 1: itching, reddening, foreign body sensation \\
\hline 2 & $\begin{array}{l}\text { Stage 2: stage } 1+\text { tearing, vasodilation of conjunctiva bulbi } \\
\text { (threshold value) }\end{array}$ \\
\hline 3 & $\begin{array}{l}\text { Stage 3: stage } 2+\text { vasodilation and erythema of conjunctiva } \\
\text { tarsi, blepharospasm }\end{array}$ \\
\hline 4 & $\begin{array}{l}\text { Stage 4: stage } 3+\text { chemosis, lid swelling (did not occurred } \\
\text { during this study) }\end{array}$ \\
\hline
\end{tabular}

Table 1: Gronemeyer's grading scale to assess allergic severity in CPT [4] Threshold value for positive CPT is 2 .

*Corresponding author: Anatoli Astvatsatourov, ${ }^{1}$ Institute of Medical Statistics,Informatics and Epidemiology University Hospital Cologne Lindenburger Allee 42, Cologne, Germany, Tel: 81-859-22-6856; E-mail: anatoli.astvatsatourov@uni-koeln.de

Received April 24, 2014; Accepted July 28, 2014; Published July 31, 2014

Citation: Astvatsatourov A, Mosges R (2014) Euclidean Norm in Composite Severity Score to Evaluate an Allergic Reaction from Conjunctival Provocations. J Health Med Informat 5: 159. doi:10.4172/2157-7420.1000159

Copyright: (c) 2014 Astvatsatourov A, et al. This is an open-access article distributed under the terms of the Creative Commons Attribution License, which permits unrestricted use, distribution, and reproduction in any medium, provided the original author and source are credited. 
Citation: Astvatsatourov A, Mosges R (2014) Euclidean Norm in Composite Severity Score to Evaluate an Allergic Reaction from Conjunctival Provocations. J Health Med Informat 5: 159. doi:10.4172/2157-7420.1000159

Page 2 of 3

\begin{tabular}{|c|c|c|c|c|c|c|c|c|c|c|c|}
\hline \multicolumn{4}{|c|}{$n=1$} & \multicolumn{4}{|c|}{$n=2$} & \multicolumn{4}{|c|}{$n=3$} \\
\hline$s_{1}$ & $s_{2}$ & $s_{3}$ & $C_{j}$ & $s_{1}$ & $s_{2}$ & $s_{3}$ & $C_{j}$ & $s_{1}$ & $s_{2}$ & $s_{3}$ & $C_{j}$ \\
\hline 4 & - & - & 4,00 & 1 & 4 & - & 1,03 & 1 & 1 & 4 & 0,47 \\
\hline 3 & - & - & 3,00 & 0 & 4 & - & 1,00 & 0 & 1 & 4 & 0,46 \\
\hline 2 & - & - & 2,00 & 1 & 3 & - & 0,79 & 0 & 0 & 4 & 0,44 \\
\hline & & & & 0 & 3 & - & 0,75 & 1 & 1 & 3 & 0,37 \\
\hline & & & & 1 & 2 & - & 0,56 & 0 & 1 & 3 & 0,35 \\
\hline & & & & 0 & 2 & - & 0,50 & 0 & 0 & 3 & 0,33 \\
\hline & & & & & & & & 1 & 1 & 2 & 0,27 \\
\hline & & & & & & & & 0 & 1 & 2 & 0,25 \\
\hline & & & & & & & & 0 & 0 & 2 & 0,22 \\
\hline & & & & & & & & 1 & 1 & 1 & 0,19 \\
\hline & & & & & & & & 0 & 1 & 1 & 0,16 \\
\hline & & & & & & & & 0 & 0 & 1 & 0,11 \\
\hline
\end{tabular}

Table 2: Composite score $C$ of the $j$-th patient, calculated by Equation 1 for all possible combinations of the assessed severity scores, according to the grading conditions, used in the described study.

divided by the square number of challenges $n^{2}\left(n=i\right.$, if $\left.s_{i} \geq 2\right)$ :

$$
C_{j}=\frac{\sqrt{\sum_{i=1}^{n} s_{i}^{2}}}{n^{2}}
$$

At a maximum of three challenges, at $0 \leq s_{i} \leq 4$ and at $s_{t h}=2$, Equation 1 conforms to the following two requirements:

- Each combination of the $s_{i}$ results in a unique $C_{j}$.

- The decreasing $\mathrm{C}_{j}$ value indicates an improvement of allergic severity (hyposensitisation). The $C_{j}$, calculated for all possible $0 \leq \mathrm{s}_{i} \leq 4$ with $\mathrm{s}_{\mathrm{th}}=2$ and $1 \leq n \leq 3$, are presented in Table 2 .

A mean of the composite score at "time- $\mathrm{x}$ " for a group of $\mathrm{N}(\mathrm{tx})$ patients is:

$$
\bar{C}(t x)=\sum_{j=1}^{N(t x)} C_{j}(t x) / N(t x) .
$$

The change of the mean composite score between "time-0" and "time-1" represents the therapeutic effect:

$$
\Delta \bar{C}=\bar{C}(t 0)-\bar{C}(\mathrm{t} 1)
$$

The number of patients participating in the CPT study at "time- 1 " $\mathrm{N}(t 1)$ can be smaller than the number of patients at "time- 0 " $\mathrm{N}(\mathrm{t} 0)$ if someone drops out before the visit at "time-1". To avoid bias only patients tested both at "time-0" and at "time-1" must be considered, i.e. $N(\mathrm{t} x)$ in Equation 2 must be $N(\mathrm{t} 0) \cap N(\mathrm{t} 1) \equiv N(t 1)$. However, the number of challenges can change for some patients at "time-1" in a positive or negative direction. In order to estimate fluctuations $\phi$ induced by changes in the number of challenges for some patients, the following procedure is suggested:

1 The difference of the single score is calculated for each $j^{*}$-th patient:

$$
\Delta s_{i, j^{*}}=s_{i, j^{*}}^{(t 0)}-s_{i, j *}^{(t 1)}
$$

Here, $j^{*}$ denotes those patients, who were tested both at "time-0" and at "time- 1 ", i.e. $1 \leq j^{*} \leq N(t 1)$.
2 The value $\Delta_{\mathrm{Cj} *}$ is introduced for each $j^{*}$-th patient. It is calculated as an Euclidean norm of $\Delta s_{i, j *}$ divided by the square minimal number of challenges $n^{* 2}, n^{*}=\min \left(n_{(t))}, n_{(11)}\right)$, taking into account the sign of each $\Delta \mathrm{s}_{\mathrm{i}, j *}$ :

$$
\begin{gathered}
\widehat{S}=\sum_{i=1}^{n^{*}} \operatorname{sgn}\left(\Delta s_{i, j^{*}}\right) \times \Delta s_{i, j^{*}}^{2} \\
\Delta c_{j *}=\operatorname{sgn}(\widehat{\mathrm{S}}) \times \sqrt{|\widehat{S}|} / n^{* 2}
\end{gathered}
$$

3 For a group of patients $N(\mathrm{t} 1)$ the arithmetic mean of the $\Delta_{C_{j^{*}} \text {-values is: }}$

$$
\bar{\Delta}_{C}=\frac{\sum_{j^{*}=1}^{N(t 1)} \Delta_{C_{j^{*}}}}{N(t 1)}
$$

4 The maximal fluctuation $\phi_{\max }$ is defined as an absolute difference of values calculated in Equations 3 and 4:

$$
\phi_{\max }=\left|\bar{\Delta}_{C}-\Delta \bar{C}\right|
$$

\section{Results}

The therapeutic effect obtained in a randomized controlled doubleblind dose-finding study of 159 patients with birch pollen allergy is presented in Figure 1. Here, quantitative values of the triangle data points, connected with a solid line, are calculated by Equations 1-3 for 150 patients, which have participated all study visits. The triangles show the therapeutic effect, as a change in the mean composite score $\Delta \bar{C}$, for four groups of patients treated with the medicament in four different doses M1-M4, respectively. The dots connected with the dashed line in Figure 1 indicate the qualitative success of the therapy for the M1-M4 treatment groups. This qualitative estimation represents the normalized number of patients in each M1-M4 group, whose symptoms are improved. The normalization factor is $123(82 \%$ of 150)-number of patients with improved symptoms in all treatment groups. The CPT was carried out before and after the treatment. Error bars of the quantitative values, shown by the triangle points represent statistical errors of the mean and the error bars of the qualitative dot 


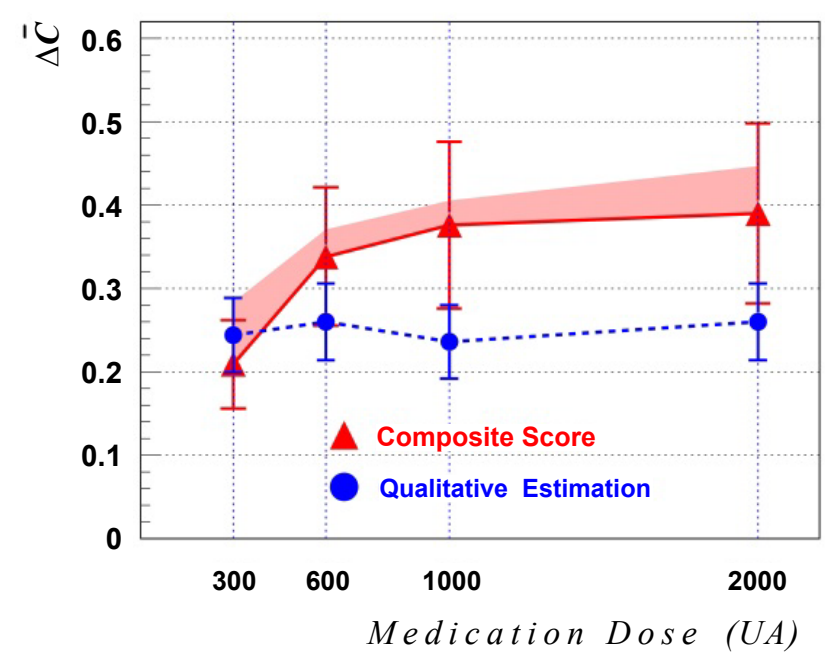

Figure 1: Therapeutic effect $(\Delta \bar{C})$, calculated by Equations 1,2 and 3 for four patients groups, each of which was treated with one of the medication doses M1-M4 is shown by triangles, connected with solid line. Error bars represent statistical errors of the mean. The dots connected with dashed line represent the qualitative estimation of the therapeutic effect for patients with improved symptoms. Error bars represent a square root of number of patients in the corresponding dose group.

points-the square root of the number of patients in the corresponding M1-M4 group.

The $\bar{\Delta}_{C}$ value, calculated by Equation 4 , is shown as the upper limit of the shadowed area. The shadowed area represents the fluctuation $\phi$ with the maximal value $\phi_{\max }$ of the Equation 5. The fluctuation is induced by the difference of $N(t 1)$ from $N(t 0)$ and by changes in the number of challenges at "time- 0 " and "time-1". The improvement of the allergic reaction increases from the treatment dosage M1 to M3 where $\Delta \bar{C}$ reaches a plateau and remains on a fairly constant level. To determine the statistical significance the $\mathrm{U}$-test is used. The significance level of $\alpha=5 \%$ for the therapy effect is not reached in the assessment of the difference between the dosage groups. The obtained $\mathrm{p}$-value in the U-test is less than 0.1 .

\section{Discussion}

Comparison of the composite score values (triangles in Figure 1) with the qualitative values (dots in Figure 1) shows a clear advantage of the technique, which is sensitive to all scores and to the number of applied solutions. However, the described method is novel in the allergic severity assessments and requires the validation, which is planned to be done in the coming studies, including assessments of the test-retest reliability, convergent and discriminant validity.

A change in the CPT solution doses or in the dilution ratio should not affect the form of the therapeutic effect distribution, which is supposed to have a logarithmic behavior. However, the statistical effects can affect the distribution.

\section{Summary}

The developed method for calculating a composite score of several individual measurements, each resulting in a single score $0 \leq s_{i} \leq 4$, with the number of measurements restricted to $3(i=1,2,3)$, was successfully applied in the estimation of the therapeutic effect in this dose-finding study in allergy. The method is planned to be used as standard in analysis of the next clinical studies in allegry with conjuctival provocation.

\section{Acknowledgements}

The authors express deep thanks to Ms. Marie-Josefine Joisten and Dr. Alexander Henke for very useful remarks and suggestions.

\section{References}

1. Peshkin MM (1931) A dry pollen ophthalmic test in pollen asthma and hay-fever patient negative to cutaneous tests. J Allergy 3: 20-29.

2. Loveless $M H$ (1945) The conjunctival test as a guide to clinical clinical immunity in hay-fever. Ann Allergy 3: 333-340.

3. Moller C, Bjorksten B, Nilsson G, Dreborg S (1984) The precision of the conjunctival provocation test. Allergy 39: 37-41.

4. Riechelmann H, Epple B, Gropper G (2003) Comparison of conjunctival and nasal provocation test in allergic rhinitis to house dust mite. Int Arch Allergy Immunol 130: 51-59.

5. Klimek L, Schendzielorz P, Pinol R, Pfaar O (2012) Specific subcutaneous immunotherapy with recombinant grass pollen allergens: first randomized dose-ranging safety study. Clin Exp Allergy 42: 936-945.

6. Klimek L, Willers J, Hammann-Haenni A, Pfaar O, Stocker H, et al. (2011) Assessment of clinical efficacy of CYT003-QbG10 in patients with allergic rhinoconjunctivitis: a phase Ilb study. Clin Exp Allergy 41: 1305-1312.

7. Gronemeyer U (1988) Konjunktivaler provokations test. In: Praktische Allergologie. Thieme Stuttgart 67-68. 